\title{
CIRCULATORY FAILURE IN ACUTE INFECTIONS
}

\author{
By RICHARD V. EBERT AND EUGENE A. STEAD, JR. \\ (From the Medical Clinic of the Peter Bent Brigham Hospital and the Department of Medicine, \\ Harvard Medical School, Boston)
}

(Received for publication June 27, 1941)

Failure of the circulation in acute infectious diseases produces a clinical picture that resembles in many ways that seen in hemorrhage, traumatic shock, or nitrite collapse. The patients show narrowing of the field of consciousness, pallor, sweating, cold extremities, rapid feeble pulse, collapsed veins, and low arterial pressure. The failure of the circulation in hemorrhage and traumatic shock has been shown to be caused by a diminished blood volume. Transfusion of blood or plasma, therefore, restores the circulation. In collapse which occurs in subjects in the upright position after the ingestion of sodium nitrite, circulatory failure is caused by pooling of blood in dilated veins and venules in the lower part of the body. Hence, returning the subject to the horizontal position restores the circulation. The purpose of this paper is to describe the changes in the circulation which occur in the so-called "medical shock" produced by certain acute infections, and to determine whether a diminished blood volume or venous pooling is the primary factor in the production of this type of circulatory failure.

\section{METHOD}

Before concluding that a diminished blood volume is of primary importance in producing a given type of circulatory failure, it is necessary to demonstrate $(a)$ that the blood volume is decreased, and $(b)$ that the circulatory failure will not occur if the decrease in blood volume is prevented. In previous experiments on an unselected group of dying patients with terminal circulatory failure, a decrease in plasma volume was frequently found (1). From this data alone it was not possible to determine whether the change in plasma volume was really a significant factor in the circulatory failure, or whether it was merely an incidental finding secondary to the disease process itself, or to an inadequate fluid intake or to malnutrition. In the present study, therefore, more emphasis has been placed on the response of the circulation to transfusion than on the measurement of the plasma volume. In studying the response of the circulation to transfusion, it was essential to demonstrate that the volume of blood had been increased effectively, and that fluid had not left the blood stream as fast as it was given. This was done in two ways: (1) by following the hematocrit reading, the serum protein concentration, and the plasma volume, and (2) by administering fluid until the pressure was increased in the large veins. The plasma volume was determined by the method of Gibson and Evans (2), as adapted to the photoelectric colorimeter (3). A $1.6 \mathrm{per}$ cent solution of potassium oxalate was used for the hematocrit determinations. The serum protein determinations were performed by the falling drop method of Kagan (4). The hemoglobin concentration was measured with the photoelectric colorimeter (5) or by the method of Sahli. Venous pressure measurements were made by the method of Moritz and von Tabora (6). The femoral or external jugular veins were used in most cases, as it is difficult to obtain an accurate venous pressure measurement from the arm veins when there is marked peripheral vasoconstriction. Arterial pressures were determined by the use of a mercury manometer and auscultation or palpation.

\section{OBSERVATIONS}

Eight cases of circulatory failure in acute infectious diseases were studied (Table I). There were 6 cases of pneumococcus pneumonia, one case of hemolytic streptococcus septicemia complicating a urethral stricture and chronic pyelonephritis, and one case of staphylococcus septicemia. Six of the 8 cases had a bacteremia. All the patients studied presented a similar clinical picture. They were stuporous or comatose. The rectal temperatures ranged from $97^{\circ}$ to $106.4^{\circ} \mathrm{F}$. The skin was pale and often covered with perspiration. The extremities were cold, and this finding usually preceded the fall in arterial pressure. The skin of the body was usually warm, although in the terminal stages it too became cool. The radial pulse was feeble or impalpable. The pulsations in the femoral artery were more prominent than those in the radial artery. In the patients with pneumonia the respirations were usually rapid and deep, and tracheal râles were often present. The respirations were normal in Case 7 who had hemolytic streptococcus septicemia.

In all the cases included in the study, there was a fall in arterial pressure. The systolic and dias- 


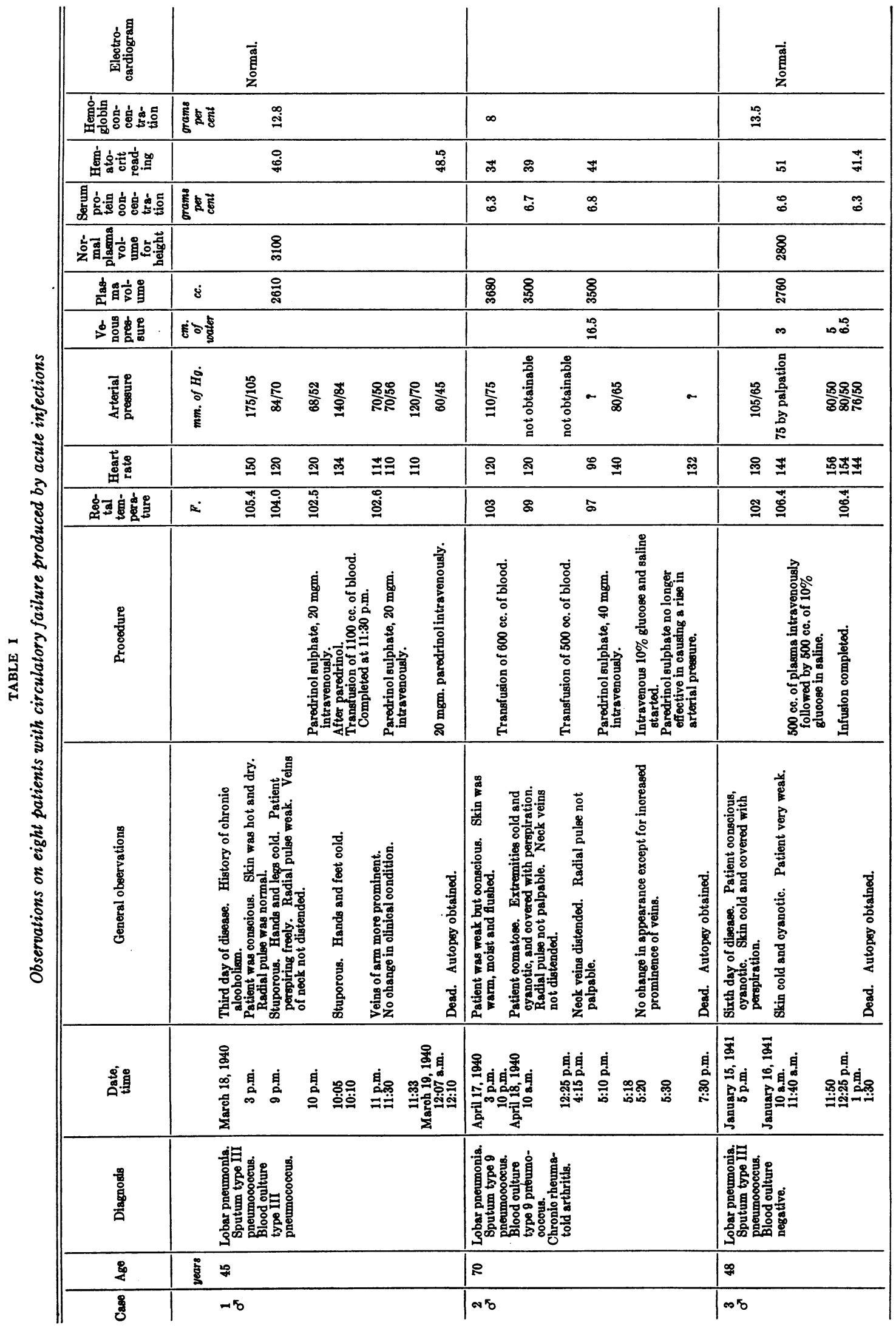




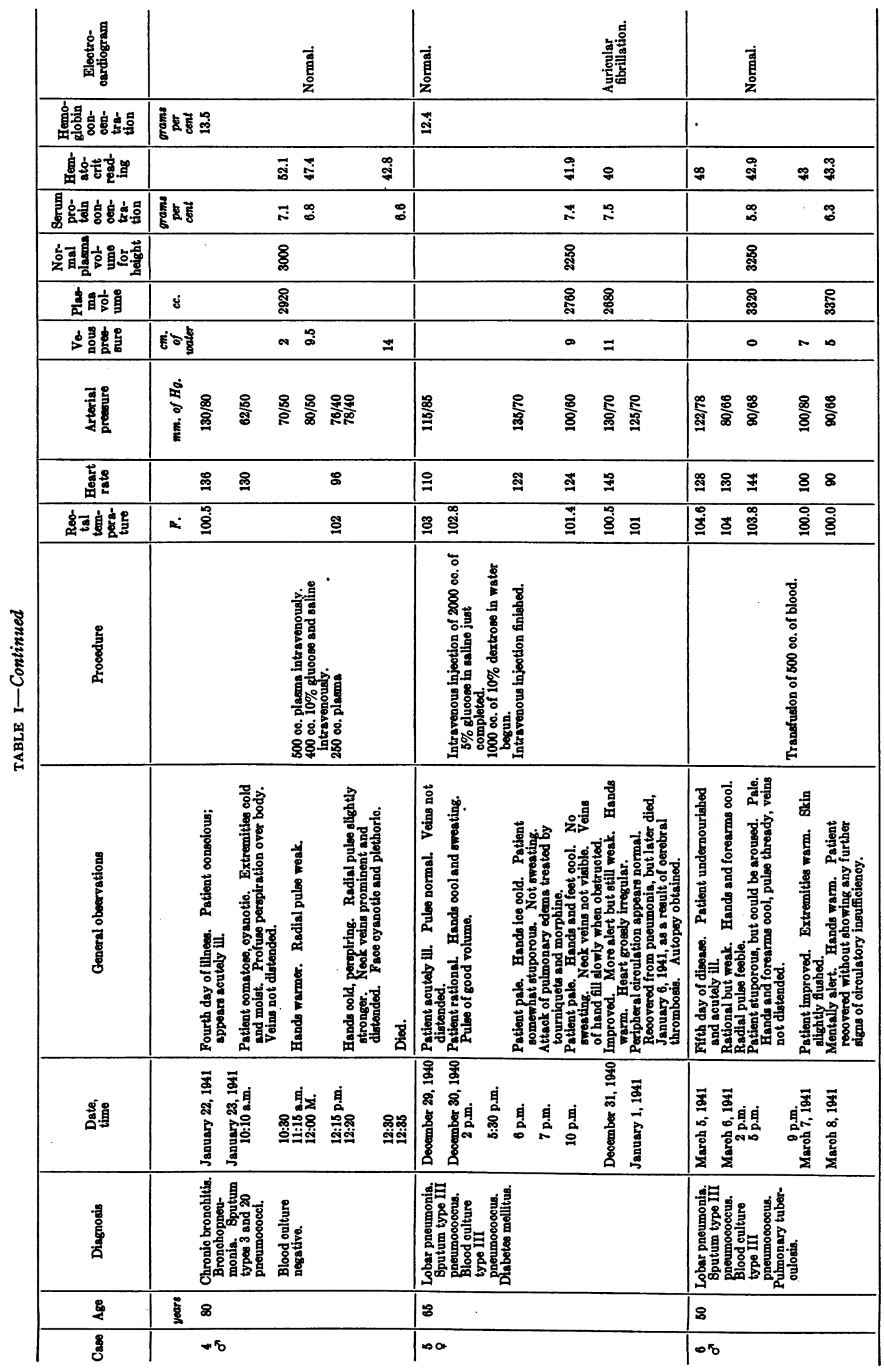




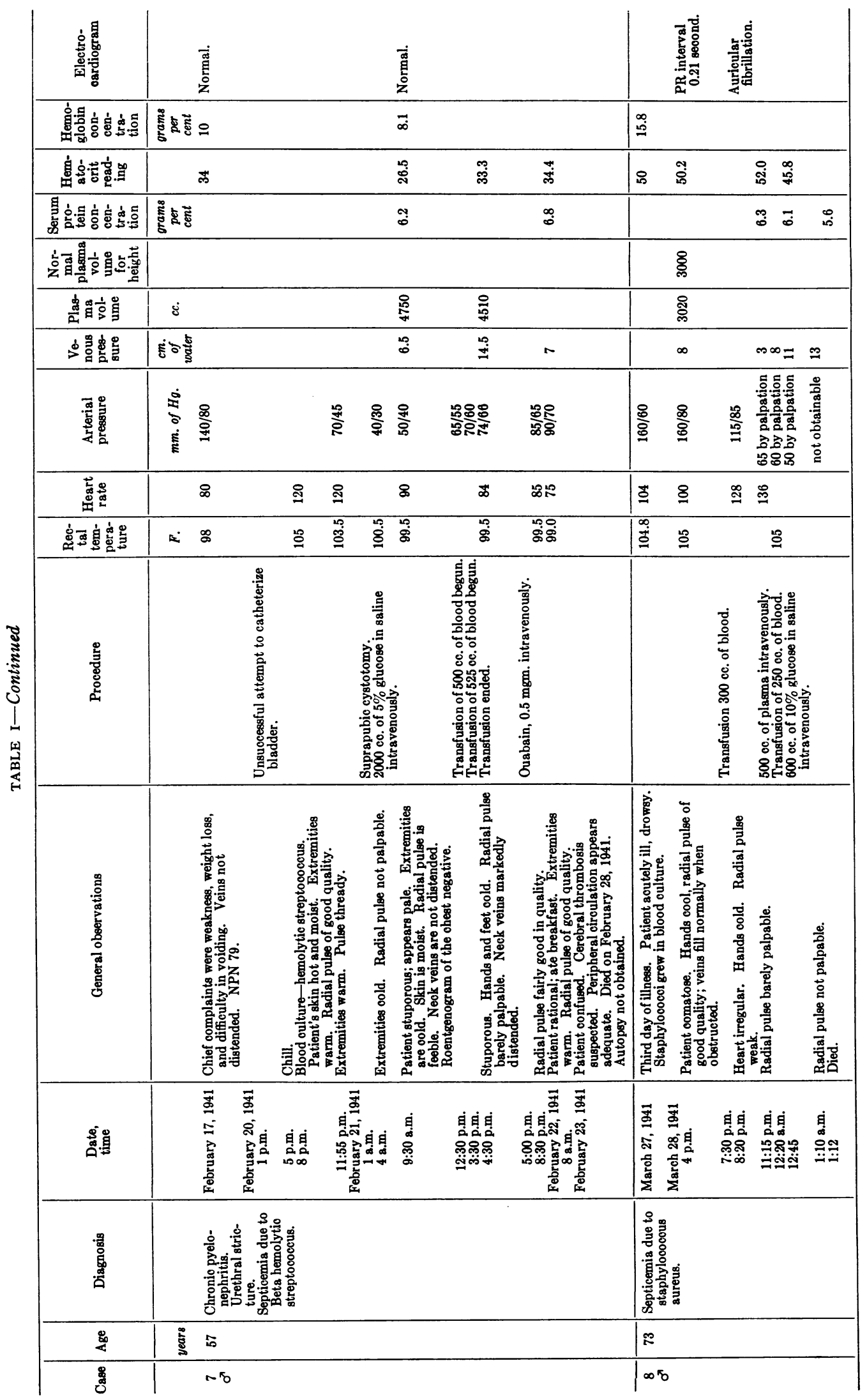


tolic pressures both decreased and the pulse pressure became narrower. The fall in arterial pressure was often marked in degree, and in some cases the arterial pressure could not be obtained either by auscultation or palpation in the later stages of the circulatory failure. The pulse rate was rapid. Two cases developed auricular fibrillation during the period of circulatory failure. The venous pressure was determined before the administration of blood or plasma in 5 cases. The values ranged from 0 to $8 \mathrm{~cm}$. of water. Thus the venous pressure was not elevated in any of these cases. Electrocardiograms were normal in 5 cases, and showed auricular fibrillation in 2 cases.

Marked hemoconcentration was not present in any of the cases studied. The hematocrit readings before the administration of blood or plasma ranged from 26.5 to 52.1 , and the serum protein concentrations ranged from 5.8 to 7.4 grams per $100 \mathrm{cc}$. Plasma volume determinations were made in all cases and showed no significant variation from the normal. In the 6 cases in which no significant anemia was present, the plasma volume determined before the administration of blood or plasma averaged 0.3 per cent above the normal value for the patient's height, as given by Gibson and Evans (2). The values for the plasma volume ranged from -19 to +22 per cent of the normal value for the height. In Case 2 the plasma volume determined before the onset of the circulatory failure, when the blood pressure was $110 / 75$, did not differ significantly from the plasma volume determined when the patient had severe circulatory failure and the blood pressure could not be obtained. In Case 5, in whom temporary recovery occurred, and in Case 6 in whom permanent recovery occurred, there was no significant change in plasma volume after improvement of the circulation.

The effect of change in posture on the arterial pressure was determined in 4 cases. The arterial pressure was determined in the horizontal position, in the Trendelenburg position with the foot of the bed raised 18 inches off the floor, and with the patient sitting at an angle of 50 degrees. In Cases 3,6 and 7, there was no difference in the arterial pressure. in the different positions. In Case 4, the arterial pressure was $62 / 50$ in the sitting position and $70 / 50$ in the Trendelenburg position.
The effect of transfusions of blood or plasma on the arterial pressure and clinical condition was determined in 6 cases. Four patients (Cases 1, 2, 7 , and 8) received 1000 to $1100 \mathrm{cc}$. of blood or plasma. One patient (Case 4) received $750 \mathrm{cc}$. of plasma, and another patient (Case 3 ) received 500 cc. of plasma. In addition, several patients were given a solution of 10 per cent glucose in saline intravenously (Table I). In none of the patients was there any definite improvement immediately after the administration of the blood or plasma. Case 7 improved temporarily, but this improvement did not begin until several hours after the transfusion. The effect of transfusions on arterial pressure was slight. In 2 patients there was no change in arterial pressure after transfusion. In 3 patients there was a rise in systolic arterial pressure of 5 to $10 \mathrm{~mm}$. of mercury. In one patient the arterial pressure continued to fall during the transfusion. In 4 patients the venous pressures before and after transfusion were compared. An increase in venous pressure occurred in all subjects. The increase ranged from $3 \frac{1}{2}$ to $8 \mathrm{~cm}$. of water and averaged $7 \mathrm{~cm}$. of water. In one patient (Case 2), the venous pressure was measured only after transfusion. In this patient the venous pressure appeared clinically to be normal before transfusion. After transfusion, clinically it appeared elevated and measured $161 / 2 \mathrm{~cm}$. of water.

In the 3 patients receiving plasma (Cases 3,4 , and 8 ), there was a lowering of the hematocrit reading after the administration of the plasma, indicating that an increase in plasma volume had occurred. In 2 patients who received whole blood (Cases 2 and 7), plasma volume and hematocrit readings were determined after transfusion. In these cases there was a slight decrease in plasma volume and a moderate increase in hematocrit reading. In persons with an adequate circulation, similar changes occurred after transfusion.

Two patients in the study (Cases 1 and 2) were given paredrinol sulphate intravenously. In both cases there was a striking rise in arterial pressure which lasted approximately 20 minutes. In these patients the radial pulse became stronger, but their general conditions appeared the same. In both cases injections of paredrinol sulphate, given shortly before death, had no effect. These 
observations show that at a time when the circulation was not benefited by transfusion, it was still able to respond to an adequate stimulus.

The ulnar nerve at the elbow was injected with novocain in Cases 2 and 8 . At the time of injection, the extremities were cold, the radial pulse was thready or impalpable, and the blood pressure had begun to fall. In each subject the ulnar side of the hand, the little and ring fingers became warmer than the other fingers of the same hand or than the fingers of the opposite hand. The difference in temperature was unmistakable and was confirmed by several observers who did not know that the ulnar nerve had been injected.

\section{DISCUSSION}

All the patients selected for study had circulatory failure characterized not only by a marked fall in arterial pressure, but also by a decrease in peripheral blood flow as shown by pallor, cold extremities and collapsed veins. A fall in arterial pressure alone cannot be used as a criterion for selecting cases of circulatory failure, because in many instances a moderate decrease in arterial pressure may occur without any signs of circulatory insufficiency; in rarer cases, even a striking fall in arterial pressure may not be accompanied by a great decrease in peripheral blood flow. It must also be remembered that in many patients dying of acute infection, the circulation is adequate but death results from other causes, such as respiratory failure or aspiration of vomitus.

Failure of the circulation of the type described here is most commonly seen in overwhelming infection associated with bacteremia. It occurs most frequently in the older age groups or in persons who are poorly nourished because of chronic disease or inadequate intake of food.

Patients with acute infection and circulatory failure of the type described here clinically resemble in many ways cases of traumatic shock or hemorrhage. In both there are signs and symptoms of diminished peripheral blood flow and tissue anoxia. For this reason it has been suggested that circulatory failure in acute infections, in traumatic shock, and in hemorrhage, has the same etiology-namely, a diminished blood volume (7, 8, and 9). Eppinger and Schurmeyer (7) stated that the circulating blood volume, as measured by the carbon monoxide method, is decreased in shock associated with acute infectious diseases. They attributed the circulatory failure to a decreased venous return to the heart. Andrews and Harkins (9) weighed the lungs in patients dying of pneumonia, and suggested that the circulatory failure resulted from the loss of plasma into the lungs with a consequent decrease in plasma volume. The data reported here indicate that the circulatory failure seen in acute infections differs in two essential ways from that of hemorrhage or traumatic shock; (a) laboratory studies do not show any evidence of a diminished blood volume or of hemoconcentration, and $(b)$ transfusions are not an effective form of therapy. It must be remembered that in the cases studied here, the fluid intake was maintained either by mouth or by the parenteral administration of fluid. Undoubtedly, in patients with diarrhea or vomiting who have not received sufficient parenteral fluid, dehydration and hemoconcentration may play an important part in producing circulatory insufficiency, and the administration of fluid will cause improvement. This paper is concerned with patients in whom circulatory failure has occurred in spite of an adequate fluid intake.

It has been suggested that failure of the vasomotor center is responsible for the circulatory failure in acute infectious diseases (10). Investigation in experimental animals has shown that the vasomotor center continues to function in circulatory failure caused by hemorrhage and traumatic shock (11). Porter (12) concluded from his investigations in animals that the vasomotor center functions normally in acute infections. In the cases reported here, the development of cold extremities in the presence of a high rectal temperature and before a marked fall in arterial pressure had occurred indicates that there was a diminution in peripheral blood flow. That this decrease in blood flow was due in part to neurogenic vasoconstriction was demonstrated in Cases 2 and 8 by the fact that the ulnar side of the hand and the little finger became warmer after neurogenic impulses were removed by block of the ulnar nerve at the elbow. While these experiments show that vasoconstrictor impulses were reaching the vessels of the hand, they do not prove that the functions of the vasomotor center were necessarily normal. 
It has been thought that the circulatory failure in acute infections may be the result of pooling of blood in dilated capillaries and veins, so that the venous return to the heart becomes inadequate $(13,14)$. This type of circulatory failure can be produced in the laboratory by motionless standing. Because of gravity, the blood accumulates in the dependent portions of the body, the venous return to the heart becomes inadequate, and the circulation fails. When the subject is placed in the horizontal or Trendelenburg position, blood flows back from the dilated capillaries and venules, the venous return to the heart becomes adequate, and the circulation rapidly returns to normal. In the cases studied here, the failure of the circulation to improve in the Trendelenburg position indicates that the circulatory failure was not the result of pooling of blood in capillaries and venules which could be drained towards the heart by elevation of the lower portion of the body. It might still be argued that the blood was trapped in the smaller vessels and that even with the aid of gravity the blood could not reach the great veins, where gravity would be effective in increasing the venous return to the heart. That this was not the case was shown by transfusion of blood and plasma and by infusion of 10 per cent glucose in saline. Adding fluid to the vascular bed of sufficient amount to cause distension of the superficial veins and an average rise in pressure of $7 \mathrm{~cm}$. of water. in the femoral or external jugular veins did not cause the circulation to improve significantly. In these cases there was no question of trapping blood in the periphery.

Although the data reported here indicate that peripheral pooling of blood is not the primary mechanism in producing circulatory failure, there is considerable evidence that the tone of the small blood vessels is altered. Acute respiratory infections are known to produce postural fainting, presumably through a loss of venous tone (15). Similar observations have been made in patients with pneumonia (16). Other investigators (17, 18) have studied the tone of the small vessels of the skin in pneumonia by measuring the height to which the venous pressure must be raised to obliterate an area of blanching produced by pricking epinephrine into the skin. They concluded that the tone of the small vessels of the skin is decreased.
In recent years, little emphasis has been placed on the heart as a factor in circulatory failure in acute infectious diseases because of the absence of the classical picture of congestive failure and because of the ineffectiveness of digitalis therapy (19). Certain experiments on animals also served to draw attention away from the heart. Romberg, Passler and others (10) demonstrated in rabbits with acute infections that the arterial pressure could be raised by pressure on the abdomen or by the administration of saline solution intravenously. From this they concluded that the heart was functioning normally. Newburgh and Porter (20) found that the hearts of dogs with pneumonia would contract normally when removed from the body. The fact that in the patients reported here the addition of fluid to the vascular system caused an average rise in venous pressure of $7 \mathrm{~cm}$. of water without causing significant improvement in the circulation indicates that the heart was not functioning normally. None of the patients had an elevation of the systemic venous pressure before transfusion. In cases of pneumonia it is difficult to evaluate pulmonary congestion but, in the patient with streptococcus septicemia (Case 7), the roentgenogram of the lung showed no evidence of congestion. The fact that auricular fibrillation developed in 2 cases also suggests that the infection had a deleterious effect on the myocardium. The absence of venous congestion, however, demonstrates that other factors must also play an important rôle in this type of circulatory failure.

The data suggest that circulatory failure in acute infections is not produced by the failure of a single portion of the cardiovascular system, but the entire cardiovascular system appears to be damaged by the infection. It is unlikely that the lack of response to transfusions is due to permanent irreversible damage to the circulatory system secondary to the fall in arterial pressure. In Case 7 , there was no significant response to transfusions although, when the infection subsided, a period of temporary improvement occurred. In the other patients studied, the observations were made shortly after the fall in arterial pressure began. From the experimental data it is impossible to state the exact sequence of events in circulatory failure in acute infections. A working hypothesis which is in accord with observed facts is as fol- 
lows: The cardiac output falls because of damage to cardiac function, and compensatory vasoconstriction occurs in the arterioles. The tone of the veins and venules is decreased, and therefore the venous pressure is not elevated.

The only effective form of therapy in these cases has been that directed toward controlling the infection. In Case 7 in whom the infection subsided spontaneously, a period of temporary improvement occurred. In Case 6, in whom the infection was combated with sulfathiazole and large doses of antipneumococcal serum, the signs of circulatory failure disappeared. The same was true of Case 5, although this patient later died of a cerebral thrombosis which probably developed during the period of circulatory failure. Efforts directed towards the treatment of circulatory failure itself resulted in no significant improvement.

\section{SUMMARY AND CONCLUSIONS}

1. Eight patients with circulatory failure produced by acute infection were studied. There were 5 cases of lobar pneumonia, 4 of which had bacteremia. There was 1 case of streptococcal septicemia, 1 of staphylococcal septicemia, and 1 of bronchopneumonia without bacteremia. The circulatory failure was characterized by a decrease in peripheral blood flow and a fall in arterial pressure.

2. Measurements of the hematocrit level, the serum protein concentration, and the plasma volume, showed no evidence of significant hemoconcentration or of a diminished blood volume.

3. The venous pressure determined before transfusion was normal.

4. Elevating the foot of the bed did not improve the circulation.

5. Transfusions of whole blood, or plasma, or the infusion of 10 per cent glucose in saline until the venous pressure rose, did not produce any improvement in the circulation.

6. Blocking the ulnar nerve caused the ulnar side of the hand and the 4th and 5th fingers to become warmer than the other fingers. This showed that the vasoconstriction in the hand was neurogenic in origin.

7. The circulatory failure in these cases does not have the same mechanism as that of hemorrhage or traumatic shock, because the plasma volume is not decreased and transfusions are not beneficial.
It is not caused by venous pooling, because filling the venous system does not improve the circulation.

8. The entire cardiovascular system appears to be damaged by the infection. The absence of congestion, and the fact that the venous pressure is not increased, may be explained by simultaneous injury to the heart and loss of venous tone.

9. Improvement in the circulation occurs only when the infection is brought under control. Therapy should therefore be directed towards overcoming the infection rather than attempting to treat the circulatory failure itself.

This investigation was carried out with the technical assistance of Miss Rosamond Piotti.

The authors wish to thank Dr. Maxwell Finland, Boston City Hospital, for allowing them to make observations on two patients. Part of the plasma used in this study was provided through the courtesy of Dr. Max M. Strumia, Bryn Mawr Hospital, Bryn Mawr, Pa.

\section{BIBLIOGRAPHY}

1. Stead, E. A., Unpublished data.

2. Gibson, J. G., 2nd, and Evans, W. A., Clinical studies of the blood volume. I. Clinical application of a method employing the azo dye "Evans blue" and the spectrophotometer. J. Clin. Invest., 1937, 16, 301.

3. Gibson, J. G., 2nd, and Evelyn, K. A., Clinical studies of the blood volume. IV. Adaptation of the method to the photoelectric microcolorimeter. J. Clin. Invest., 1938, 17, 153.

4. Kagan, B. M., Simple method for estimation of total protein content of plasma and serum. II. Estimation of total protein content of human plasma and serum by use of falling drop method. J. Clin. Invest., 1938, 17, 373.

5. Evelyn, K. A., Stabilized photoelectric colorimeter with light filters. J. Biol. Chem., 1936, 115, 63.

6. Moritz, F., and von Tabora, D., Uber eine Methode, beim Menschen den Druck in ober flächlichen Venen exakt zu bestimmen. Deutsches Arch. f. klin. med., 1909, 98, 475.

7. Eppinger, H., and Schurmeyer, A., Uber den Kollaps und analoge zustände. Klin. Wchnschr., 1928, 7, 777.

8. Moon, V. H., Shock and Related Capillary Phenomena. Oxford University Press, New York, 1938.

9. Andrews, E., and Harkins, H. N., "Surgical shock" factors in pneumonia. Ann. Int. Med., 1937, 10, 1503.

10. Romberg, E., and others, Untersuchungen über die allgemeine Pathologie und Therapie der Kreislaufstörung bei acuten Infectionskrankheiten. Deutsches Arch. f. klin. med., 1899, 64, 652.

11. Seelig, G., and Joseph, D. R., On the condition of the vaso-constrictor center during the development of shock. J. Lab. and Clin. Med., 1916, 1, 283. 
12. Porter, W. T., Newburgh, L. H., and Newburgh, I., The state of the vasomotor apparatus in pneumonia. Am. J. Physiol., 1914, 35, 1.

13. Atchley, D. W., Medical shock. J. A. M. A., 1930, 95, 385.

14. Warfield, L. M., Treatment of circulatory failure. J. A. M. A., 1936, 106, 892.

15. Weiss, S., Unpublished data.

16. Stead, E. A., Jr., and Ebert, R. V., The peripheral circulation in acute infectious diseases. Med. Clin. of North America, 1940, 24, 1387.
17. Perry, C. B., Peripheral circulation in acute lobar pneumonia. Quart. J. Med., 1934, N. S., 3, 273.

18. Greene, D., Rôle of peripheral circulation in pneumonia in children. Am. J. Dis. Child., 1937, 53, 947.

19. Niles, W. L., and Wyckoff, J., Studies concerning digitalis therapy in lobar pneumonia. Am. J. M. Sc., 1930, 180, 348.

20. Newburgh, L. H., and Porter, W. T., The heart muscle in pneumonia. J. Exper. Med., 1915, 22, 123. 